\title{
The use of human neurons for novel drug discovery in dementia research
}

\section{Mariana Vargas-Caballero, Sandrine Willaime-Morawek, Diego Gomez- Nicola, V. Hugh Perry, Diederik Bulters \& Amrit Mudher}

To cite this article: Mariana Vargas-Caballero, Sandrine Willaime-Morawek, Diego GomezNicola, V. Hugh Perry, Diederik Bulters \& Amrit Mudher (2016): The use of human neurons for novel drug discovery in dementia research, Expert Opinion on Drug Discovery, DOI: 10.1517/17460441.2016.1154528

To link to this article: http://dx.doi.org/10.1517/17460441.2016.1154528

Accepted author version posted online: 15

Feb 2016.

Submit your article to this journal $\pi$

Џ Article views: 15

Q View related articles $\sqsubset$

View Crossmark data $\nearrow$ 
Publisher: Taylor \& Francis

Journal: Expert Opinion on Drug Discovery

DOI: $10.1517 / 17460441.2016 .1154528$

\section{Review:}

The use of human neurons for novel drug discovery in dementia research

\section{Mariana Vargas-Caballero ${ }^{*}, 1,2$, Sandrine Willaime-Morawek ${ }^{3}$, Diego Gomez-} Nicola $^{1}$, V. Hugh Perry ${ }^{1}$, Diederik Bulters ${ }^{*}, 4$, Amrit Mudher ${ }^{*}, 1$.

( ${ }^{*}$ corresponding authors)

University of Southampton

1. Centre for Biological Sciences,

2. Institute for Life Sciences

3. Clinical Neurosciences and Psychiatry, Faculty of Medicine and Centre for Human Development, Stem Cells and Regeneration; University of Southampton,

4. Wessex Neurological Centre

\section{Dr Mariana Vargas-Caballero}

Centre for Biological Sciences, University of Southampton, B85 Highfield Campus, Southampton, SO17 1BJ

m.vargas-caballero@soton.ac.uk

\section{Dr. Sandrine Willaime-Morawek}

Clinical Neurosciences and Psychiatry, Faculty of Medicine and

Centre for Human Development, Stem Cells and Regeneration; University of Southampton,

South Laboratory Block, Southampton General Hospital, Mailpoint 840, Southampton SO16 6YD

S.Willaime-Morawek@soton.ac.uk

\section{Dr Diego Gomez-Nicola,}

Centre for Biological Sciences, University of Southampton,

LD80C, South Laboratory Block, Southampton General Hospital, Mailpoint 840, Southampton SO16 6YD

\section{D.Gomez-Nicola@soton.ac.uk}

\section{Professor V. Hugh Perry,}

Centre for Biological Sciences, University of Southampton,

LD80, South Laboratory Block, Southampton General Hospital, Mailpoint 840, Southampton SO16 6YD

v.h.perry@soton.ac.uk 


\title{
Mr Diederik Bulters,
}

Consultant Neurosurgeon,

Wessex Neurological Centre, Southampton General Hospital

Southampton SO16 6YD

dbulters@nhs.net

\section{Dr Amrit Mudher}

Centre for Biological Sciences, University of Southampton, B85 Highfield Campus, Southampton, SO17 1BJ

a.mudher@soton.ac.uk

\section{Abbreviations used:}

New Molecular Entities (NMEs)

Embryonic stem cells (ESC)

Induced pluripotent stem cells (iPSC)

Human induced pluripotent stem cells (hiPSCs)

Post mortem (PM)

\begin{abstract}
.
Introduction. Although many disease models exist for neurodegenerative disease, the translation of basic research findings to clinic is very limited. Studies using freshly resected human brain tissue, commonly discarded from neurosurgical procedures, should complement on-going work using stem cell-derived human neurons and glia thus increasing the likelihood of success in clinical trials.
\end{abstract}

Areas covered. Herein, the authors discuss key issues in the lack of translation from basic research to clinic. They also review the evidence that human neurons, both freshly resected brain tissue and stem cell-derived neurons, such as induced pluripotent stem cells (iPSCs), can be used for analysis of physiological and molecular mechanisms in health and disease. Furthermore, the authors compare and contrast studies using live human brain tissue and studies using induced human stem cellderived neuron models. Using an example from the area of neurodegeneration, the authors suggest that replicating elements of research findings from animals and stem cell models in resected human brain tissue would strengthen our understanding of disease mechanisms and the therapeutic strategies and aid translation. 
Expert opinion. The use of human brain tissue alongside iPSC-derived neural models can validate molecular mechanisms identified in rodent disease models and strengthen their relevance to humans. If drug target engagement and mechanism of cellular action can be validated in human brain tissue, this will increase the success rate in clinical research. The combined use of resected human brain tissue, alongside iPSC-derived neural models, could be considered a standard step in pre-clinical research and help to bridge the gap to clinical trials.

\section{Article highlights}

- $\quad$ Fewer than $10 \%$ of new drugs entering clinical trials achieve licensing for human use and the biggest risk of failure is in Phase II trials.

- Confirming that a new drug binds the target and modifies its function can increase confidence in its likelihood to succeed in Phase II clinical trials.

- Human neuron experimental paradigms in which new drugs could be tested include stem-cell models and resected brain tissue typícally discarded from neurosurgical procedures.

- $\quad$ iPSC-derived neurons can be obtained from patients and have great potential for drug testing.

- $\quad$ Resected adult human brain tissue can help scientists to establish benchmarks for further development of iPSC-derived neural cultures and validate findings from iPSC-derived neurons, thus bridging the gap between pre-clinical and clinical research.

- $\quad$ Analysis of tau protein complexity in mature human neurons in physiological conditions will clarify its role in disease and may contribute to targeting it therapeutically. 


\section{Introduction}

Research into diverse brain disorders has relied on the use of post-mortem tissue and experimental models to understand the cellular and molecular mechanisms of disease. Such experiments have been invaluable in expanding our understanding of the function of neurons and glia as well as how different diseases affect them. The lack of translation from pre-clinical research to patient benefit is therefore surprising. As a solution to this problem, the drug discovery field is moving towards experimental paradigms using human neurons. Currently these models are based on stem cellderived neurons and to a lesser degree the use of residual human brain tissue derived from neurosurgical procedures. We discuss a) some key issues that may be responsible for lack of translation from pre-clinical to clinical research; b) current experimental approaches using human neurons that we believe can provide better translation. We provide key examples to illustrate our opinions and one focused example of how these issues may have contributed to a lack of translation for treating dementia. We provide suggestions on how the different experimental paradigms using human neurons can be used in a complementary manner to facilitate translation in drug discovery.

\section{Problems with translating knowledge of brain diseases into drug therapies.}

Whether from the perspective of a large pharmaceutical company or that of a patient, the data on translation of new candidate drugs from pre-clinical research to achieving licensing for human use is startling. Although the exact figures are debated, the general message is clear. Across all indications $65 \%$ of agents are successful in Phase 1 clinical trials, 32\% in Phase 2, 60\% in Phase 3, of which $83 \%$ achieve eventual licensing. This equates to $10 \%$ of agents entering Phase 1 resulting in a clinically used drug ${ }^{1,2}$. The situation for New Molecular Entities (NMEs) (7.5\%) and neurological indications (9.4\%) is even worse. Given the costs associated with clinical trials these figures have attracted enormous attention with extensive analysis of how this may be improved. 
To date, attention has mostly focused on the inadequacies of pre-clinical animal testing and clinical trial design. It is clear both can be improved. There is no doubt that current animal models of disease need better validation and that more rigorous experimental design would reduce noise in experimental observations ${ }^{3}$. Furthermore, higher quality reporting of animal experiments is urgently needed to ensure reproducibility of data as stated in the ARRIVE (Animal Research: Reporting of In Vivo Experiments) guidelines initiative ${ }^{4}$.

Similarly, clinical trials need to be refined. Seemingly simple issues such as changes in endpoints between early and late phase studies or problems generalizing results from narrow research indications to the wider population remain practically complex and challenging ${ }^{5}$.

However, much of the problem lies in the statistics. Whereas in pre-clinical research, models are designed to be as homogeneous and reproducible as possible in an attempt to control for all other factors, real diseases and patient groups are intrinsically highly heterogeneous. This heterogeneity means that the numbers of subjects studied, often single or double digits in pre-clinical studies, quickly become three or four digits long in clinical studies (and despite this may remain underpowered).

This is just one juncture at which the powering of a study is against us. For a new agent to be licensed multiple hypotheses will have been tested in successive studies, each with its own challenges. Moreover, our illusion is that if we achieve a $p$ value under 0.05 in any one study we should be on solid ground for progression as our agent only has a $5 \%$ chance of failure at the next stage of testing. However this assumes that the candidate agent is clinically safe and effective. It has been argued that, if only $10 \%$ of new candidate drugs are suitable for use, then if studies are conventionally reported with a significance of $5 \%$ and power of $80 \%$ there will be a $4.5 \%$ false positive and $8 \%$ true positive rate. In essence only $56 \%$ of "statistically significant" results are likely to be true positives. This is compounded by many early studies not meeting these power thresholds and reporting multiple outcome measures, which has led some to suggest that as few as 30\% of statistically significant results are true positives ${ }^{6}$. The obvious response to this is to reset the level of significance as has already been suggested ${ }^{7}$. However this ignores the real world practicalities and costs 
of the populations required for such studies, particularly for some of the rarer indications.

In reality a solution must lie in improving the selection of candidate drugs put forward for clinical testing. However, with the high throughput screening programmes that have become increasingly available in the laboratory more new agents are considered for more indications than ever. Multiple testing is subject to the same statistical considerations as the clinical trials discussed earlier and thus rather than ameliorating the problem it compounds it. This reinforces the need for more extensive selection procedures for candidate drugs put forward for clinical testing, which may be achieved with appropriate preclinical testing in more and better models. It will additionally require cross validation between laboratories and publication of negative results.

All the above arguments apply to drug discovery in general. The case is even stronger for neurological disorders. This is also where the limitations of using animals are greatest as analysing cellular and molecular mechanisms in the brain requires invasive studies. It is after all the complexity of the human brain that sets humans aside from animals. Indeed many animal models of disease do not fully recapitulate the psychiatric and neurodegenerative conditions being investigated ${ }^{8}$, and models can be quite removed from the clinical reality.

What can we learn from clinical trials to improve the success rate in drug development? Morgan et al performed a meta analysis on Phase II decisions for 44 programs at Pfizer ${ }^{9}$ to understand the fundamental knowledge that can increase success rate in clinical trials. They concluded that success in Phase II clinical trials depended on an integrated understanding of "exposure at the site of action (I), target binding (II) and expression of functional pharmacological activity (III)". They termed these three elements: the 'three pillars of survival'. Pillars II and III increase the pharmacological confidence in the NME. To translate the knowledge from in vitro or animal work the NME must bind to the target and the functional molecular mechanisms downstream of drug action must be engaged in humans; thereby accounting for potential impact of species differences and discrepancies in the translation of research findings from rodents to humans. 
What could improve experimental models to increase success rates in Phase II trials? The use of in vitro human tissue and human cells has the potential to have a significant impact as it has been demonstrated in oncology by the use of fresh human tumour tissue to test new therapeutics on specific human tumours and tumour cell lines ${ }^{10}$.

Use of post-mortem brain tissue has increased markedly with the development of brain banks to facilitate access to tissue ${ }^{11}$ and has been invaluable in improving our understanding of disease. However, this tissue does not contain live cells to test physiological and pharmacological mechanisms. In addition, post-mortem delay may affect key aspects of protein biology including post-translational modifications, cellular localisation and the extent of protein degradation.

These factors can be controlled for when using fresh human brain tissue derived from neurosurgical procedures. During neurosurgery there are some instances where normal brain is removed to obtain access to a lesion underlying it such as an epileptic focus, brain tumour, vascular anomaly or haemorrhage. In many instances this tissue is routinely discarded, but if it is collected in theatre and rapidly processed in physiological media, it remains healthy and electrophysiologically active for up to 24 hours ${ }^{12}$. It can provide an invaluable an insight into the structure and function of adult human neurons and glia $^{13-15}$. It is therefore surprising that with such a valuable and very limited resource, only a small fraction of what is routinely removed at surgery is currently used for physiological and pharmacological testing. This is where live brain tissue is of greatest benefit for translational research.

Resected human brain tissue is limited in availability and can be cultured for only a few weeks ${ }^{16}$. For long term in vitro culture and high throughput screening of candidate drugs another human neuron experimental paradigm is needed. One rapidly growing approach is to utilise human stem cell derived neurons generated either from embryonic stem cells (ESCs) or from induced pluripotent stem cells (iPSCs) ${ }^{17}$. iPSCs are adult cells -such as skin fibroblasts- that have been reprogrammed to an embryonic-like state and that can be then differentiated into specific cell types by distinct protocols. However, it remains to be proven how accurately iPSC-derived neurons reflect fully mature neurons. 
Some benchmarks of neuronal structure and function have been derived from our extensive knowledge of rodent neurons, and are already routinely applied to human iPSC (hiPSC) cultures. For example, expression and subcellular localisation of markers such as PSD-95, synaptotagmin, synapsin1 and vglut1 are used to address maturity. However, we know that in many aspects hiPSCs are relatively immature compared to adult human neurons. The input resistance of cortical neurons, a measure of neuron size and active membrane channels, is almost two orders of magnitude different between hiPSCs $(2000-5000 \mathrm{M} \Omega)^{18}$, and rodent (64 M $\Omega$ ) or human $\left(86 \mathrm{M} \Omega\right.$ ) neurons ${ }^{12}$. Furthermore, recent studies have shown that adult human neurons are indeed different in many properties to mouse neurons. Cortical human neurons are three times larger with far more complex dendritic trees than rodent neurons ${ }^{14}$, and these differences in their morphology are mirrored by differences in the molecular composition of synapses ${ }^{19}$ and glial components ${ }^{13}$ producing complex physiological properties that impact on signal integration ${ }^{20}$. Therefore the gold standard against which to compare hiPSCs-derived neurons and glia should be cells in adult human brain tissue.

We propose that studies in resected human brain tissue can significantly complement existing drug development programs. There are clearly a number of challenges that this poses, but these are not insurmountable. If resected human tissue is used routinely for this purpose then there will be greater translation of knowledge from animal models to novel drug discovery.

\section{How can live human brain tissue be used for understanding mechanisms of human brain disease.}

Resected human brain tissue can be obtained from neurosurgical procedures once appropriate ethical permission has been put in place. This type of tissue is already being used with great success to analyse synaptic integration ${ }^{20}$ and synaptic plasticity $^{12}$ in cortical neurons, and to understand the mechanism of action of neuroactive drugs ${ }^{15}$. Although human brain samples available to the research community are highly heterogeneous, it is possible to analyse properties that can be generalised across different conditions. We suggest that a detailed analysis of the 
tissue with a degree of standarisation will permit data sharing across different institutions and will allow us to confirm and replicate findings about neuronal physiology, disease mechanisms, and drug actions on therapeutic targets.

3.1 Dealing with the diversity of tissue collected from neurosurgical cases for analysis of neuronal function in health and disease. The pathological tissue resected during neurosurgery can be used to understand the underlying disease. Epileptic cortical tissue from patients with drug-resistant epilepsy was used to understand oscillatory mechanisms, showing consistent results across a range of primary pathologies ${ }^{21}$. However, a proportion of the tissue derived from neurosurgical cases can be classified as "non-pathological" by means of imaging, macroscopic and molecular examination. This tissue can be used as a tool in drug discovery for diseases other than the disease that necessitated neurosurgery.

To date the most commonly used human brain tissue has been derived from patients with epilepsy. While tumour surgery is far more frequent, only a small proportion of cases yield normal tissue, and although sections are smaller it has also been utilised as has tissue from patients with vascular malformations. Tissue availability remains limited and is highly dependent on the pathologies considered and local neurosurgical practices. Overall, one might expect 10 to 40 neurosurgery cases per year yielding resected brain tissue in a typical neurosurgical unit (of which there are about 25 in the United Kingdom).

Since access to this precious resource is limited when compared to other experimental approaches such as in vitro or in vivo animal studies, data-sharing across laboratories engaged in using human brain tissue can be a powerful strategy to generate a large data-base and repeat observations. In order to facilitate this, data annotation becomes of paramount importance, owing to the variety of neurosurgical procedures, specific sites of tissue origin, and the diversity of case characteristics such as patient age, sex, co-morbidities, prescription drugs, and so forth. A public database, such as CARMEN, allows data-sharing ${ }^{22}$, with highly standarised reporting requirements ${ }^{23}$.

In addition, given that the non-pathological tissue originates from a brain with a neurological condition, albeit derived distally from pathology focus, it becomes 
necessary to establish its degree of normality. This can be achieved by generalising across samples from patient groups with unrelated neurological conditions. Indeed Verhoog et al. were able to reproduce experimental observations analyzing the mechanisms of synaptic plasticity in samples derived from both epilepsy patients and cancer patients ${ }^{12}$.

A number of criteria are already in use by different groups to evaluate the pathology in resected human tissue and many of these measures can be combined for screening the tissue. The results can be annotated in the corresponding database with additional image files containing immunohistochemistry or western blot data where available. Experimental approaches for characterising tissue include:

The pre-surgical imaging and in situ examination needs to show no signs of structural anomalies, including bleeding, or hyper/hypo-intensities. Additionally, the distance of origin of resected tissue from the pathological focus should be reported.

- The activation of microglia and astrocytes is a good sensor of pathological anomalies, as these cells react quickly to changes in the microenvironment ${ }^{24}$. Staining the resected tissue for the expression of markers of activation of microglia (i.e. MHCII, iNOS) or astrocytes (i.e. Vimentin) would indicate the level of pathology, as these markers are not usually expressed by resting cells. Classical markers such as Iba1 (microglia) or GFAP (astrocytes) can also be used to detect changes in cell morphology (gliosis), characteristic of glial activation.

For samples obtained from epileptic patients, several techniques have been used to analyse the extent of epileptic activation on resected tissue outside the epileptic focus: - Expression of the phosphorylated forms of CREB and/or ERK, have been proposed as neuronal biomarkers of epilepsy ${ }^{25,26}$ using either Western blot analysis or immunohistochemistry; the latter can provide a spatial correlate with recorded neurons.

Resected samples need to show reduced or low levels of neuronal expression of the immediate early gene transcription factors EGR-1, EGR-2, and c-fos, as these have been shown to dominate the gene expression pattern of epilepsy cases ${ }^{26}$.

Analysis of pathological tissue from the different patient groups will provide a contrast and control for the health of the resected tissue under study. 
The qualitative and quantitative observations of the above variables will provide a good measure of quality of the resected tissue, and should be annotated along with other experimental data for subsequent analysis.

\subsection{Why use human brain tissue instead of rodent brain tissue? Although animal} models have played a critical role in our understanding of neuronal function in health and disease, there are significant differences between the rodent and human brain. These are not only related to brain size and differences in total numbers of neurons ${ }^{27}$ but also differences at the molecular and cellular level that we are just beginning to fully characterise. For example, layer 3 (L3) cortical pyramidal neurons in human have double the density of postsynaptic spines compared to equivalent mouse neurons ${ }^{28}$. Furthermore, approximately $30 \%$ of the proteins in excitatory synapses in human are not present in mouse synapses, and there are substantial differences in the abundance of postsynaptic proteins that are expressed in these species ${ }^{19}$. There are differences at the level of protein isoform expression: there are only three isoforms of tau protein expressed in adult mice while there are six isoforms in adult human. It is likely that some of these differences have contributed to failure of translation of neuroactive compounds in pre-clinical to clinical work.

Some of the key features of synaptic transmission originally discovered in animal models have now been confirmed in human neurons, such as synaptic plasticity: the capacity of neurons to strengthen or weaken their connections following specific patterns of stimulation 29,30 . However, recent work has suggested that physiological and morphological properties of human neurons endow them with distinct computational rules in the processing of neuronal signals when compared with rodents $^{12,14,31}$ Human neurons are able to recover much faster from short-term depression. This property arises from differences in cellular morphology; human L2/L3 pyramidal neurons have 3 times more dendrites than their mouse and macaque counterparts- imposing distinct electrical properties ${ }^{14}$ and endowing them with up to ten times the capacity for information transfer.

\subsection{Extending the use of resected brain tissue via organotypic slice culture. Although} resected human brain tissue is remarkably resilient, allowing for high quality recordings for up to 24 hours after resection ${ }^{12}$, it would be desirable to further extend 
the lifetime of these samples. This would enhance data collection for electrophysiology experiments and would permit the analysis of cellular variables that require longer time scales, such as changes in mRNA or protein expression, following acute treatments with drugs or other manipulations including molecular techniques to express transgenes in specific cell populations, for example allowing the analysis of neuronal function using optogenetics.

A number of laboratories have already analysed resected human brain tissue under organotypic culture conditions and have shown that such tissue can indeed be maintained in culture for 3-4 weeks ${ }^{16,32,33}$. This work highlights an advantage of human brain organotypic culture: the ability to culture mature brain sections. Rodent organotypic cultures can only be obtained from immature animals, typically less than 2 weeks of age. The inability to culture rodent adult brain tissue has imposed a problem on the analysis of phenotypes that arise in mature animals such as development of plaques/tangles in transgenic models but there are some recent insights into the underlying changes in adult mouse brain tissue that prevent long term organotypic culture. Mewes et $\mathrm{al}^{33}$ performed a metabolic and immunohistochemical analysis of murine brain slices that had been maintained in culture comparing slices from neonatal vs. adult animals. They showed that slices from neonatal mice maintained a high degree of metabolic vitality for weeks in culture while slices from 7-10 month old transgenic mice decreased in vitality and showed deteriorating neuronal morphology, activation of astrocytes and microglia, and programmed cell death ${ }^{34}$. Another study showed that it may indeed be possible to culture hippocampal slices from 3-5 month old adult mice if a serum-free medium is used ${ }^{35}$. Under these conditions many tissue characteristics are well preserved such as neuronal morphology, however a sharp decrease in synaptic proteins such as synapsin and PSD-95 is initially observed at 7 days in vitro with stable levels maintained thereafter. Although a qualitative analysis suggests that human neurons can maintain their morphology in organotypic culture ${ }^{16}$, quantitative analyses of vitality, content of synaptic proteins and glial activation as described above for mouse slices have not been carried out. Performing such characterisation in organotypic cultures of resected human brain will further inform and validate this approach to extend the lifetime and experimental tools available for this type of tissue. 
The use of brain tissue derived from neurosurgery can help us understand the function of neuronal circuits and identify therapeutic targets. Although the tissue will be derived from patients undergoing different types of neurosurgery, the tissue can be subjected to thorough characterisation. Tissue can be analysed under control conditions or following acute treatments that mimic disease, along with treatments and could provide proof of principle for drug actions. The ability to perform both control and experimental analyses in samples from the same patient will add statistical power to studies allowing for paired analysis. Being able to confirm target engagement and drug mechanisms in samples from distinct patients has great potential to increase the pharmacological confidence in compounds to then proceed to trials and test in individuals.

The practicalities in obtaining resected human brain tissue and its analysis may not, however, allow for high throughput experiments or long-term culture of all brain regions. It is here that the study of human neurons derived from hiPSCs would provide a complementary approach with many advantages, as discussed below.

\section{The use of stem cell models in drug discovery, how far have we come characterising them and using them to understand disease?}

Human stem cells can be reprogrammed to generate differentiated neurons and glia in vitro. These stem cells can be obtained from embryos (hESC) or from adult human cells, which have been reprogrammed to achieve a stem cell potential (hiPSC). The advantage of the models using human hiPSCs is the fact that these cells can be derived from adults, and indeed the patients themselves. Thus, they capture the whole genotype associated with a disease, without focussing solely on one or more mutations identified for that particular disease. In contrast to human tissue resected from neurosurgical cases, hiPSCs can be cultured for months, thus allowing long-term experimentation and observation including analysis under chronic drug treatments. Numerous studies have used hiPSCs to model either normal neuronal development and maturation, or pathological conditions. This experimental paradigm is very attractive for creating translatable models of neurological diseases but it poses many challenges ${ }^{36}$ including the difficulty in reproducing the right cell types at the required 
stage of maturity in the appropriate microenvironment and cells that recapitulate a disease phenotype. Although there are many examples of models that have successfully addressed these challenges, there are still some elements that need to be developed further ${ }^{17}$ such as cellular diversity, network connectivity and neuronal maturity. We discuss some of these challenges below but recognise that this is a very fast evolving field and tremendous progress is being made to overcome these problems.

4.1 Variability and Reproducibility. There is high variability within a single hiPSCs line, due to passaging and accumulation of mutations, as well as variability between cell lines derived from the same patient ${ }^{37,38}$. Not all the cell lines have the same differentiation potential, and several lines from a single patient are often needed to get a highly neurogenic line. This experimental variability within a patient is compounded by the variability between patients where genome wide and epigenetic changes cause the disease phenotype. It will be important to understand how these factors interact to give rise to the disease and to extract those elements that have a greater effect size. Reproducibility of findings across multiple hiRSC lines from as many different patients as possible is important here to confirm the relevance of findings to the disease phenotype. However, there may be financial and time limitations to analyse many different cell lines from different patients. Instead we believe that it might be possible to obtain a higher number of replications in comparative experiments using adult human neurons derived from neurosurgery, where cell line associated variability is not a factor. If a disease phenotype can be acutely mimicked in the resected tissue, or the molecular/drug mechanism of action can be confirmed using this paradigm, this would greatly strengthen the experimental observations from the hiPSC models and increase confidence in the therapeutic target in human.

4.2 Differentiation protocols. Numerous studies have used hiPSCs to model aspects of neurodegenerative disease using different methods of differentiation to recapitulate some features of the disease, as recently reviewed ${ }^{39}$. Protocols exist for generating specific neuronal subtypes including cholinergic neurons ${ }^{40-42}$, dopaminergic neurons ${ }^{38}$ and glutamatergic neurons ${ }^{43}$. However, differentiation protocols are highly diverse and as recently reviewed ${ }^{38}$, stem-cell derived models of Parkinson's disease, employ a variety of differentiation protocols in which $4 \%-37 \%$ of neurons acquire dopaminergic 
marker tyrosine hydroxylase after anything from 21 to 70 days of differentiation. This clearly demonstrates that specific differentiation protocols can have a significant impact on the differentiation time required to acquire a particular neuronal phenotype and the proportion of neurons committing to this phenotype. The goal of recreating every desired neuronal cell type primarily affected in the disease under study for example, entorhinal cortex and hippocampal cell types for Alzheimer's disease, has not been achieved. To date there is no protocol to differentiate pluripotent cells specifically into entorhinal cortex cells, although recent work suggests that differentiation of dentate gyrus granule cells is indeed possible owing to their intrinsic neurogenic capacity in the adult brain ${ }^{44}$. Overall, current protocols are still often complex, lengthy, expensive and yield mixed populations of neurons, constituting a challenge for analysis of specific cell subtypes.

\subsection{Maturation and aging. Many neurodegenerative diseases affect the synaptic} activity of neuronal networks in the aging brain, thus it is important to develop mature neuronal networks to allow the development of reliable disease models ${ }^{17,36}$. Existing 3D culture studies recapitulate only developing neural cells (mid-end of gestation stage) and little or no synaptic activity. Using hESCs or human neural stem cells 3D cultures have been developed and their maturity has been equated to that of a first or second trimester stage of gestation ${ }^{45}$, showing excitable neurons with spontaneous glutamatergic synaptic activity ${ }^{46,47}$ Similar results have been obtained using hiPSCs, demonstrating maturation to mid-foetal stage only ${ }^{48}$ with neurons able to fire repetitive action potentials in response to depolarising current steps and spontaneous glutamatergic synaptic transmission ${ }^{48}$. To mimic the ageing of hiPSC-derived neuronal cultures 51 , further strategies are currently under development, for example, progerin expression ${ }^{52}$.

Appropriate benchmarks are required to assess maturity and functionality of stem-cell derived neurons (as reviewed $\mathrm{in}^{53}$ ). A complete morphological and structural characterisation of neuronal maturation would include immunohistochemistry analysis of mature neuron markers such as NeuN, MAP-2, adult tau isoforms, synaptic proteins such as synapsin and PSD95, and the apposition of such pre- and post-synaptic proteins. Further characterisation by electron microscopy ${ }^{52,53}$ can provide confirmation of synapse formation. For electrophysiological characterisation, an analysis of intrinsic 
excitability, spontaneous and evoked synaptic activity is required ${ }^{53}$. The level of maturity of neurons may depend on specific differentiation and culturing protocols. $A$ recent study showed that the standard culture media used widely to culture hiPSC impairs neuronal signalling, and developed a new serum-free cell culture media which better supports neuronal activity, synaptic connectivity and survival of human neurons from hiPSCs in culture ${ }^{18}$.

All stem cell-derived neural cells for modelling neurodegenerative disease ultimately aim to recreate the structure and function of adult human neurons or glia. A direct comparison of the physiology of stem-cell derived neurons with resected adult human neurons will provide the best benchmarks to illustrate how similar stem-cell neurons are to actual adult human neurons and to what extent they differ. This knowledge will be useful for further refining stem-cell protocols to ultimately generate neurons that most closely resemble adult brain neurons in vivo.

4.4 3D cultures: 3D culture systems aim to model the physiological environment of neurons more closely by providing a diffusional barrier as compared to monolayer cultures. 3D neural cultures systems have been developed using cells other than hiPSCs. Air liquid interface systems have been used with hESCs ${ }^{54}$ achieving a dense network of neurons, astrocytes, and oligodendrocytes reaching a developing stage similar to early fetal brain. Neural tissue derived from hESCs achieved a form of selforganization reaching a layered cortex reminiscent to a developmental stage of the second trimester of human gestation ${ }^{45}$, and 3D cultures derived from neuronal precursors expressed mature neuronal markers ${ }^{55,56}$. However, there are limited examples of hiPSCS reproducing features of human cortical development and developing into cerebral organoids or self-organising into discrete domains or layers ${ }^{49,50,57}$. To provide structural support for 3D cultures, studies have explored the option of a 3D matrix on which to grow the cells and various scaffolds have been investigated using substrates softer than plastic to improve neuronal maturation ${ }^{47,58}$.

4.5 Mixed cultures. To understand mechanisms of disease, it is necessary to incorporate into hiPSC cultures non-neuronal cell types such as glia. Indeed, astrocytes promote maturation of excitability and synaptic function in neurons differentiating from hESCs ${ }^{59}$ and increase the long-term survival of neurons 
differentiated from human neural stem cells ${ }^{60}$. 3D hiPSCs-derived neural culture studies have incorporated astrocytes in their system ${ }^{54,61}$ : a major role for astrocytes in the pathogenesis of amyotrophic lateral sclerosis has been uncovered through the coculture of motor neurons and patient-iPSC-derived astrocytes ${ }^{62}$. Incorporating patientderived microglia and vascular components into hiPSC cultures would permit the analysis of interactions between these cell populations. Although this has not been achieved with patient-derived hiPSCs, a recent study incorporated these elements successfully using human embryonic stem cell-derived precursor cells ${ }^{64}$, and demonstrated its applicability to neurotoxicity studies.

Despite differentiation, maturation and variability challenges, there is no doubt that using hiPSC-derived neural models will facilitate drug discovery and new therapeutics. Seven years after the discovery of the hiPSCs technique, several studies have recapitulated features of adult mature neurons, and used them to model aspects of a variety of neurodegenerative disease ${ }^{38,43,64}$. Encouragingly, in some of these models, disease phenotypes have been rescued with pharmacological interventions. Amyloidbeta $(A \beta)$ oligomer induced cellular stress was rescued by docosahexaenoic acid in several Alzheimer's disease patient-derived hiPSC models ${ }^{65} ; \gamma$-secretase inhibitors suppressed $A \beta$ secretion in hiPSC-derived neurons generated from patients with either PS1 or PS2 mutations ${ }^{66}$; in hiPSC-derived neurons from Phelan-McDermid syndrome patients' faulty excitatory synaptic transmission was corrected by treating neurons with insulin-like growth factor $1^{67}$. Based on hiPSCs studies, several drugs are about to enter clinical trials, BMS-986168 for progressive nuclear palsy, retigabine for amyotrophic lateral sclerosis and RG7800 for spinal muscular atrophy ${ }^{68}$.

\section{Drug translation in a specific example: Alzheimer's Disease.}

5.1 Tau centric therapies for treating dementia - why haven't they delivered? Since amyloid based therapies have not to date yielded expected results in Alzheimer's disease (AD) there has been renewed interest in tau-centred disease modifying approaches. Traditionally these have been developed in animal models of disease and have attempted to counteract pathogenic changes in tau identified in post-mortem brain tissue. They include approaches to reduce tau phosphorylation ${ }^{69}$, reduce tau 
aggregation $^{70}$ and clear toxic tau oligomers and tangles ${ }^{71}$. Additionally some studies have explored the utility of microtubule stabilising agents to compensate for loss of microtubule binding function ${ }^{72}$. Though there have been reports of some success, these tau based therapeutic strategies have had disappointing clinical outcomes ${ }^{73,74}$.

One possible reason for the failure of these various tau and amyloid targeting approaches is the late diagnosis and treatment of patients in the disease time course, The microtubule stabilising agent Davunetide, for example, significantly improved memory scores when given to patients with mild cognitive impairment ${ }^{75}$ but failed when used to treat patients with advanced tauopathy ${ }^{76}$. Another contributor to the poor translation of these therapeutic strategies may be the fact that the physiological and pathological state of tau is not entirely reflected in the post-mortem studies and animal models that are used to develop them.

5.2 Studying tau biology and pathology in human post-mortem tissue paints a clear but not complete picture. Much of what we know about tau pathology in dementia has been learned from biochemical or immunohistochemical studies conducted on postmortem tissue. The earliest investigations demonstrating that tau is hyperphosphorylated and aggregated in $A D$, and is incompetent at microtubule stabilisation were biochemical investigations comparing post-mortem brain tissue from AD patients and age-matched controls ${ }^{77-79}$. These pioneering studies were complemented by highly cited histopathological staging reports showing that such tau pathologies begin in the entorhinal cortex and spread in a characteristic manner to neocortical and other brain regions as described by Braak and Braak $1995^{80}$. This "Braak Staging" is still used to categorise disease severity and invariably correlates with extent of cognitive decline $^{81}$ (and reviewed in Nelson et al 2012) ${ }^{82}$. Subsequently many studies identified sequential phosphorylation changes in pretangle and tangle bearing neurons ${ }^{83,84}$. These studies still serve as the benchmark for abnormal tau phosphorylation in the brains of both human and animal models. Collectively these studies have contributed enormously to our understanding of how tau becomes altered in disease state. Emerging newer tools have added to this wealth of knowledge by identifying even more pathological post-translational changes in tau including isomerisation ${ }^{85}$, truncation $^{86}$, SUMOylation ${ }^{87}$, acetylation and ubiquitination ${ }^{88}$. 
Whilst such post mortem (PM) studies in disease brains have served tau biologists well by providing a framework for tau's post-translational status, aggregation status, functional competence and sub-cellular localisation in disease, the equivalent studies on PM control brain may not reflect a similarly complete picture. The post-mortem interval greatly influences key aspects of tau's biochemical properties and possibly even its sub-cellular localisation. Since pathologically altered tau in disease is relatively stable, it is less likely to be greatly changed by different PM intervals, and this may be why its pathological characteristics are generally consistent across studies. The same cannot be said for tau in normal physiological control-brain conditions. A number groups demonstrated that some phosphorylation sites previously thought only to be phosphorylated in $A D$ brains, are also phosphorylated, albeit to a smaller extent, in rapidly processed biopsy brain from control subjects. These sites identified by specific monoclonal antibodies (mab) include, amongst others, ser202/205 (recognized by mabAT8), ser396/404 (recognised by mabPHF-1) and ser262/ser356 (recognized by mab12E8) ${ }^{89,90}$. They are also phosphorylated to some extent in rapidly processed foetal and adult rodent brain ${ }^{88-90}$. Phosphorylation at these "physiological" sites is very sensitive to the post-mortem interval, decaying by $50-70 \%$ within minutes of processing. Inevitably phosphorylation and other such labile physiological post-translational tau modifications would go undetected after even with relatively short post-mortem intervals in studies on control brains. Similarly, the cellular localisation of tau is generally believed to be axonal because it has traditionally been viewed as a protein primarily involved in microtubular stabilisation. Accordingly, its presence in the somato-dendritic compartment in pre-tangle and tangle bearing neurons in $A D$ is considered a pathological mislocalisation. However, if control brain slices are rapidly fixed and processed for immunohistochemistry, AT8 positive tau is evident in the somato-dendritic compartment of wild-type animals ${ }^{91}$. The localisation of phosphorylated tau in the somato-dendritic compartment is not usually detected when rodent brains are perfused for a few minutes and then fixed for immunohistochemistry ${ }^{92}$. Indeed, with more recent studies suggesting previously unknown functions of tau, such as scaffolding functions in dendritic spines (reviewed in $^{88}$ ), it is perhaps not surprising that it will be found both in the somato-dendritic as well as axonal compartment of normal healthy neurons. 
5.3 Studying tau biology and pathology in transgenic models muddies the picture. Following on from the benchmark pathological characterisation of abnormal tau in AD brain, several very successful attempts have been made to recreate this tau pathology in transgenic models ${ }^{93}$. The models expressed either wild-type ${ }^{94}$ or familial tauopathy mutant ${ }^{95}$ forms of human tau and reproduced both cognitive impairment, phosphotau/tangle pathology and progressive neurodegeneration ${ }^{96}$. More recently, these models have begun to simulate "prion-like" disease transmission across neural networks ${ }^{97}$. Collectively, these models served to underline the causative role played by abnormal tau species in tauopathies. They have made an impressive contribution to our understanding of tau-mediated dysfunction and toxicity but they also pinpoint two other aspects of tau biology and pathology, which cannot be adequately mimicked in non-primate models. One is the need for physiological expression levels: in normal human adult brain, the $3 \mathrm{R}$ and $4 \mathrm{R}$ isoforms of tau are roughly equally expressed and disruption of this equality, or over-expression of any isoform, is pathogenic as evidenced by splicing mutations resulting in tauopathy in some familial cases. However, this delicate human isoform expression ratio is invariably not represented in many transgenic models because usually one human isoform is expressed at nonphysiological levels. Additionally, in most transgenic models, there is the confounding influence of the endogenous rodent tau, which is not always considered. Another fact highlighted by rodent models of tauopathy is the requirement of all isoforms of wildtype human tau to create tangle pathology like that seen in sporadic tauopathy. This became apparent from the so-called "humanised tau mice" in which all six isoforms of human tau were expressed and led to emergence of tangle pathology ${ }^{98}$, which was conspicuously absent in previous rodent models expressing only some of the tau isoforms.

5.4 Studying tauopathies in human neurons - closer to human brain? These studies and many others (comprehensively reviewed $i^{97,99}$ ) show how tau based therapies may not have factored in normal tau physiology when designing strategies against abnormal tau pathology. Whether this has influenced their clinical outcome is not clear but it does highlight the need to study tau biology and pathology in adult human neurons, in conditions more closely reminiscent of those found in adult brain in vivo. 
Thus far, only a few studies have described tau biology and pathology in human neuron experimental models ${ }^{43,100}$. In the study by lovino et $\mathrm{al}^{43}$, hiPSC-derived neurons were generated from fibroblasts taken from patients suffering from familial frontotemporal dementia (FTDP-17). Intriguingly, many aspects of pathology observed in brains of these tauopathy patients were recreated in the experimental culture. Developmentally regulated expression of all six tau isoforms was seen with evidence of physiological phosphorylation (at the mabAT8 site) in the control cultures and pathological phosphorylation (at the mabAT100 site) in the FTDP-patient derived cultures. Additionally, tau was located both in the axonal and somato-dendritic compartments in both the control and FTDP-patient derived cultures. As discussed above, these aspects of tau biology are generally misrepresented in studies undertaken on human post-mortem brain and are only apparent in the rapidly processed human resected brain samples. Since these hiPSC-derived cultures can be rapidly processed like resected human brain tissue, they can closely replicate physiological conditions. Thus, models such as this can be used to dissect taumediated mechanisms of dysfunction and toxicity in a physiologically relevant experimental paradigm.

Overall, tau biology is most comprehensively studied in rapidly processed human neurons because studies in postmortem tissue or rodent brain may not adequately depict its expression levels, phosphorylation status, aggregation status, functional capabilities and sub-cellular localisation. Since these are all affected in tauopathies, it is important to use models in which these aspects of tau biology replicate the biology of adult human brain. Studies in non-pathological resected human brain tissue and patient iPSC derived neural models may be the best experimental paradigms for achieving this ${ }^{43,100,101}$. However since it is unlikely for $A D$ patients to undergo neurosurgery, there will be limited access to resected tissue with confirmed AD diagnosis. Therefore disease modelling, particularly for chronic neurodegenerative diseases like $A D$, will have to rely either on acute incubation of resected tissue with disease related molecules, or manipulation of human brain organotypic cultures over days $^{33}$ or potentially weeks ${ }^{16}$. 


\section{Conclusion}

In this review we have discussed factors related to poor translation of preclinical results into clinically efficacious compounds for the treatment of disorders and diseases of the CNS. There are well recognised limitations in neurodegeneration research arising from existing experimental paradigms including use of transgenic animal models and reliance on human post mortem tissue obtained in advanced stages of disease. An important opportunity has emerged to employ patient-derived neuronal models to more closely simulate cellular and molecular mechanisms underpinning human disease but there are a number of important challenges to be addressed. We propose that a wider-use of resected human tissue from neurosurgical operations should be undertaken to validate and complement findings from the in vitro stem cell models, We have highlighted, using Alzheimer's disease as an example, how research findings may be complicated by species differences and post mortem interval in experimental paradigms, and how both human resected tissue and stem cell models would work in a complimentary manner for identification of drug targets and development of efficacious disease modifying treatments.

\section{Expert opinion}

Many factors are responsible for the limitations of translation from animal models of human neurodegenerative disease to effective drug therapy in humans. We propose that inadequate simulation of human disease in animal brains and incomplete knowledge of disease-relevant processes in adult human neurons and glia in physiological conditions may be key. The tau-based therapies and experimental approaches discussed in the example above highlight gaps in our knowledge about the physiological, biochemical and other disease-related properties in the context of tauopathies.

In a bid to generate more translatable experimental paradigms, neuroscientists have made tremendous progress in developing stem cell-derived neural models from humans. The fact that these models can be generated from patients themselves makes their use particularly relevant. Indeed stem-cell derived models that successfully recapitulate aspects of disease have been generated from patients suffering from various neurodegenerative diseases including Alzheimer's, Parkinson's, 
frontotemporal dementia and others. These models have enabled dissection of disease mechanisms and some proof-of-principle drug testing, stimulating pharmaceutical interest in using them for pre-clinical drug development. Although these models have untapped potential for better understanding human disease mechanisms in a physiological experimental paradigm, we believe that their translational impact can be further enhanced by validation of findings in resected human brain tissue. As well as validation, such comparative studies will also increase pharmacological confidence in drug targets identified from stem-cell models. This is because resected human brain tissue can serve as a stand-alone paradigm for acute aspects of pre-clinical testing of neuroactive drugs. Testing in this tissue can contribute towards two of the "three pillars of survival" in Phase II clinical trials as described by Morgan et $\mathrm{al}^{8}$ : Confirmation of target binding and expression of pharmacological activity at the target site of action increases the likelihood of drug candidate survival.

We appreciate that using resected human tissue in this way may pose many challenges, including the fact that such human tissue is a limited precious resource. To overcome some of these issues we propose that there should be a closer collaboration between neurosurgeons and neuroscientists to ensure: 1) Increased access by the scientific community to live human brain tissue derived from neurosurgery, 2) Increased efficiency and efficacy in the use of such tissue, 3) Increased crosscomparison of results with adult human tissue to validate all animal models and iPSC findings. The first of these are largely practical issues of ensuring that ethical permission is obtained, and patients consented in a sensitive and appropriate fashion and there is proximity of laboratory facilities to neurosurgical theatres. The second is centred on how to optimise the use of such brain tissue through collaborative work on both acute slices and how to maximise the lifespan of tissue with well-characterised organotypic cultures. The third challenge requires that more experimental paradigms utilise human neurons alongside iPSCs to increase mechanistic and pharmacological confidence in molecular mechanisms identified in research models such as rodents, before progressing a new candidate drug to clinical testing. Such in vitro models should inform the decision process into clinical trials.

We propose that the combined use of resected human brain tissue and patient-derived stem cell neural models for drug discovery programmes will both validate drug targets 
and enable testing of drug efficacy in a physiologically relevant experimental paradigms. If this approach becomes common practice we may be able to bridge the gap between pre-clinical research and patient benefit sooner.

\section{Declaration of Interest}

The authors are supported by Alzheimer's Research UK, Alzheimer's Society, the Gerald Kerkut Trust, the Professor Fausto Iannotti Fellowship and the Medical Research Council UK. The authors have no other relevant affiliations or financial involvement with any organization or entity with a financial interest in or financial conflict with the subject matter or materials discussed in the manuscript apart from those disclosed.

\section{Bibliography}

\section{Papers of special note have been highlighted as either of interest $\left(^{*}\right)$ or of considerable interest $\left({ }^{* *}\right)$ to readers.}

1. Hay, M., Thomas, D. W., Craighead, J. L., et al. Clinical development success rates for investigational drugs. Nat. Biotechnol. 32, 40-51 (2014).

2. Marchetti, S. \& Schellens, J.H.M. The impact of FDA and EMEA guidelines on drug development in relation to Phase 0 trials. Br. J. Cancer 97, 577-581 (2007).

3. Perrin, S. Make mouse studies work. Nature 507, 423-425 (2014).

4. Kilkenny, C., Browne, W. J., Cuthill, I. C., et al. Improving Bioscience Research Reporting: The ARRIVE Guidelines for Reporting Animal Research. PLoS Biol. 8, e1000412 (2010).

5. Gladstone, D. J., Black, S. E. \& Hakim, A. M. Toward wisdom from failure: Lessons from neuroprotective stroke trials and new therapeutic directions. Stroke 33, 2123-2136 (2002).

6. Colquhoun, D. \& London, C. An investigation of the false discovery rate and the misinterpretation of $P$ values. Hazards $P$ values 1-15 (2014). doi:10.1098/rsos.140216

7. Johnson, V. E. Revised standards for statistical evidence. Proc. Natl. Acad. Sci. 110, 19313-19317 (2013).

8. LaFerla, F. M. \& Green, K. N. Animal models of Alzheimer disease. Cold Spring Harb. Perspect. Med. 2, (2012). 


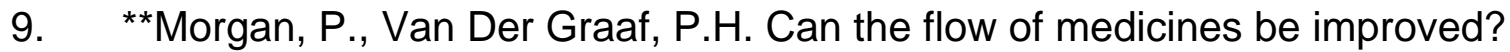
Fundamental pharmacokinetic and pharmacological principles toward improving Phase II survival. Drug Discov. Today 17, 419-424 (2012). Following an analysis of Phase II decisions at Pfizer, this article puts forward the "Three Pillars of Survival" based on an integrated understanding of the factors that impact drug candidate success in Phase II clinical trials.

10. Gillet, J.-P., Varma, S. \& Gottesman, M. M. The Clinical Relevance of Cancer Cell Lines. JNCI J. Natl. Cancer Inst. 105, 452-458 (2013).

11. UK Brain Banks Network, Medical Research Council. Available from: <http://www.mrc.ac.uk/research/facilities/brain-banks/>. [3 Feb 2016].

12. Verhoog, M. B., Goriounova, N. A., Obermayer, J., et al. Mechanisms Underlying the Rules for Associative Plasticity at Adult Human Neocortical Synapses. J. Neurosci. 33, 17197-17208 (2013).

13. Smith, A. M. \& Dragunow, M. The human side of microglia. Trends Neurosci. 37, 125-135 (2014).

14. **Mohan, H., Verhoog, M. B., Doreswamy, K. K., et al. Dendritic and Axonal Architecture of Individual Pyramidal Neurons across Layers of Adult Human Neocortex. Cereb. Cortex bhv188 (2015). Article presents a quantitative morphological analysis and computational simulations comparing cortical neurons from mice, humans and macaques. It presents sample morphologies of mouse and human cortical neurons.

15. Komlósi, G., Molnár, G., Rózsa, M., et al. Fluoxetine (prozac) and serotonin act on excitatory synaptic transmission to suppress single layer $2 / 3$ pyramidal neuron-triggered cell assemblies in the human prefrontal cortex. J. Neurosci. 32, 16369-78 (2012).

16. Eugène, E. Cluzeaud, F., Cifuentes-Díaz, C., et al. An organotypic brain slice preparation from adult patients with temporal lobe epilepsy. J. Neurosci. Methods 235, 234-244 (2014).

17. Livesey, F. J. Human stem cell models of dementia. Hum. Mol. Genet. 23, R359 (2014).

18. Bardy, C. Van Der Hurk, M., Eames T., et al. Neuronal medium that supports basic synaptic functions and activity of human neurons in vitro. Proc. Natl. Acad. Sci. 19;112(20):E2725-34 (2015).

19. **Bayés, A., Collins, M., Croning, M., et al. Comparative study of human and mouse postsynaptic proteomes finds high compositional conservation and abundance differences for key synaptic proteins. PLoS One 7, e46683 (2012). This study analyses proteome of the postsynaptic density ( 1500 proteins) and performs a comparison between mouse and human synapses. 
20. Testa-Silva, G., Verhoog, M., Linaro, D., et al. High bandwidth synaptic communication and frequency tracking in human neocortex. PLOS Biol. 12, e1002007 (2014).

21. Roopun, A. K., Simonotto, J. D., Pierce, M. L., et al. A nonsynaptic mechanism underlying interictal discharges in human epileptic neocortex. Proc. Natl. Acad. Sci. U. S. A. 107, 338-343 (2010).

22. CARMEN. Code Analysis, Repository \& Modelling for e-Neuroscience. Available from: <http://www.carmen.org.uk/>. [3 Feb 2016].

23. Gibson, F., Overton, P., Smulders, T., et al. Minimum Information about a Neuroscience Investigation (MINI): Electrophysiology. Nat. Genet. 1-8 (2008).

24. Gomez-Nicola, D. \& Perry, V. H. Microglial Dynamics and Role in the Healthy and Diseased Brain: A Paradigm of Functional Plasticity. Neurosci. 21, 169-184 (2014).

25. Beaumont, T. L., Yao, B., Shah, A., et al. CREB Target Gene Induction in Human Neocortical Epilepsy. J. Neurosci. 32, 14389-14401 (2012).

26. Rakhade, S. N., Yao, B., Ahmed, S., et al. A common pattern of persistent gene activation in human neocortical epileptic foci. Ann. Neurol. 58, 736-747 (2005).

27. Herculano-Houzel, S. The human brain in numbers: a linearly scaled-up primate brain. Front. Hum. Neurosci. 3, 1-11 (2009).

28. DeFelipe, J. The Evolution of the Brain, the Human Nature of Cortical Circuits, and Intellectual Creativity. Front. Neuroanat. 5, 1-17 (2011).

29. Chen, W. R., Lee, S., Kato, K., et al. Long-term modifications of synaptic efficacy in the human inferior and middle temporal cortex. Proc. Natl. Acad. Sci. U. S. A. 93, 8011-8015 (1996).

30. Beck, H., Goussakov, I. V, Lie, A., et al. Synaptic plasticity in the human dentate gyrus. J Neurosci 20, 7080-7086 (2000).

31. Testa-Silva, G., Verhoog, M. B., Goriounova, N. A., et al. Human synapses show a wide temporal window for spike-timing-dependent plasticity. Front. Synaptic Neurosci. 2, 2-12 (2010).

32. Verwer, R. W. H., Hermens, W., Dijkhuizen, P. A., et al. Cells in human postmortem brain tissue slices remain alive for several weeks in culture. FASEB J. 16, 54-60 (2002).

33. Sebollela, A., Freitas-Correa, L., Oliveria, F. F., et al. Amyloid- $\beta$ oligomers induce differential gene expression in adult human brain slices. J. Biol. Chem. 287, 7436-7445 (2012). 
34. Mewes, A., Franke, H. \& Singer, D. Organotypic Brain Slice Cultures of Adult Transgenic P301S Mice-A Model for Tauopathy Studies. PLoS One 7, e45017 (2012).

35. Kim, H., Kim, E., Park, M., et al. Organotypic hippocampal slice culture from the adult mouse brain: a versatile tool for translational neuropsychopharmacology. Prog. Neuropsychopharmacol. Biol. Psychiatry 41, 36-43 (2013).

36. Nityanandam, A. \& Baldwin, K. K. Advances in reprogramming-based study of neurologic disorders. Stem Cells Dev. 24, 1265-1283 (2015).

37. Sandoe, J. \& Eggan, K. Opportunities and challenges of pluripotent stem cell neurodegenerative disease models. Nat. Neurosci. 16, 780-789 (2013).

38. Badger, J. L., Cordero-Llana, O., Hartfield, E. M, et al. Parkinson's disease in a dish - Using stem cells as a molecular tool. Neuropharmacology 76 Pt A, 88-96 (2014).

39. Sproul, A. A. Being human: The role of pluripotent stem cells in regenerative medicine and humanizing Alzheimer's disease models. Mol. Aspects Med. 4344, 54-65 (2015).

40. Bissonnette, C. J., Lyass, L., Bhattacharyya, B.J., et al. The controlled generation of functional basal forebrain cholinergic neurons from human embryonic stem cells. Stem Cells 29, 802-811 (2011).

41. Crompton, L. A., Byrne, M. L., Taylor H., et al. Stepwise, non-adherent differentiation of human pluripotent stem cells to generate basal forebrain cholinergic neurons via hedgehog signaling. Stem Cell Res. 11, 1206-1221 (2013).

42. Liu, M.-L., Zhang, T., Zou, X. et al. Small molecules enable neurogenin 2 to efficiently convert human fibroblasts into cholinergic neurons. Nat. Commun. 4:2183 (2013).

43. ** Iovino, M., Agathou, S., Gonzalez-Rueda, A., et al. Early maturation and distinct tau pathology in induced pluripotent stem cell-derived neurons from patients with MAPT mutations. Brain 138(Pt 11):3345-59 (2015). This study demonstrates tau pathology in a FTDP-patient-derived stem cell model which correlates to the pathology evident in the same patient's brain post mortem.

44. Yu, D. X., Marchetto, M. C. \& Gage, F. H. How to make a hippocampal dentate gyrus granule neuron. Development 141, 2366-2375 (2014).

45. Kadoshima, T., Sakaguchi, H., Nakano, T., et al. Self-organization of axial polarity, inside-out layer pattern, and species-specific progenitor dynamics in human ES cell-derived neocortex. Proc. Natl. Acad. Sci. U. S. A. 110, 2028420289 (2013). 
46. Muguruma, K., Nishiyama, A., Kawakami, H., et al. Self-Organization of Polarized Cerebellar Tissue in 3D Culture of Human Pluripotent Stem Cells. Cell Rep. 10, 537-550 (2015).

47. Smith, I., Silveirinha, V., Stein, J., et al. Human neural stem cell-derived cultures in three-dimensional substrates form spontaneously functional neuronal networks. J. Tissue Eng. Regen. Med. (2015). doi:10.1002/term.2001

48. Mariani, J., Simonini, M. V., Palejev, D., et al. Modeling human cortical development in vitro using induced pluripotent stem cells. Proc. Natl. Acad. Sci. U. S. A. 109, 12770-5 (2012).

49. Lancaster, M. A., Renner, M., Martin, C.-A., et al. Cerebral organoids model human brain development and microcephaly. Nature 501, 373-9 (2013).

50. Paşca, A. M., Sloan, S. A., Clarke, L. E., et al. Functional cortical neurons and astrocytes from human pluripotent stem cells in 3D culture. Nat. Methods 12, (2015).

51. Sterneckert, J. L., Reinhardt, P. \& Schöler, H. R. Investigating human disease using stem cell models. Nat. Rev. Genet. 15, 625-639 (2014).

52. Miller, J. D., Ganat, Y. M., Kishinevsky, S, et al. Human iPSC-based modeling of late-onset disease via progerin-induced aging. Cell Stem Cell 13, 691-705 (2013).

53. *Bradford, A. B. \& McNutt, P. M. Importance of being Nernst: Synaptic activity and functional relevance in stem cell-derived neurons. World J. Stem Cells 7, 899-921 (2015). Comprehensive review of specific functional readouts to characterise and evaluate successful synaptogenesis in stem cell-derived neural cultures.

54. Preynat-Seauve, O., Suter, D. M., Tirefort, D., et al. Development of Human Nervous Tissue upon Differentiation of Embryonic Stem Cells in ThreeDimensional Culture. Stem Cells 27, 509-520 (2009).

55. Choi, S. H., Kim, Y. H., Hebisch, M., et al. A three-dimensional human neural cell culture model of Alzheimer's disease. Nature 515, 274-8 (2014).

56. Kim, Y.H., Choi, S. H., D'Avanzo, C., et al. A 3D human neural cell culture system for modeling Alzheimer's disease. Nat. Protoc. 10, 985-1006 (2015).

57. Lancaster, M. A. \& Knoblich, J. A. Generation of cerebral organoids from human pluripotent stem cells. Nat. Protoc. 9, 2329-2340 (2014).

58. Teixeira, A. I. Ilkhanizadeh, S., Wigenius, J., et al. The promotion of neuronal maturation on soft substrates. Biomaterials 30, 4567-72 (2009).

59. Johnson, M. A., Weick, J. P., Pearce, R. A., et al. Functional Neural Development from Human Embryonic Stem Cells: Accelerated Synaptic Activity via Astrocyte Coculture. J. Neurosci. 27, 3069-3077 (2007). 
60. Jordan, P. M., Cain, L. D. \& Wu, P. Astrocytes enhance long-term survival of cholinergic neurons differentiated from human fetal neural stem cells. $J$. Neurosci. Res. 86, 35-47 (2008).

61. Zhang, D., Pekkanen-Mattila, M., Shahsavani, M., et al. A 3D Alzheimer's disease culture model and the induction of P21-activated kinase mediated sensing in iPSC derived neurons. Biomaterials 35, 1420-1428 (2014).

62. Haidet-Phillips, A. M., Hester, M., Miranda, C., et al. Astrocytes from familial and sporadic ALS patients are toxic to motor neurons. Nat. Biotechnol. 29, 824-8 (2011).

63. *Schwartz, M. P., Hou, Z., Propson, N., et al. Human pluripotent stem cellderived neural constructs for predicting neural toxicity. Proc Natl Acad Scí US A. 112, 2-7 (2015). This work describes 3D neural cultures-derived from human pluripotent stem cells incorporating microglial and endothelial components.

64. Shi, Y., Kirwan, P., Smith, J., et al. Human cerebral cortex development from pluripotent stem cells to functional excitatory synapses. Nat. Publ. Gr. 15, 477486 (2012).

65. Kondo, T., Asai, M.,Tsukita, K., et al. Modeling Alzheimer's disease with iPSCs reveals stress phenotypes associated with intracellular $A \beta$ and differential drug responsiveness. Cell Stem Cell 12, 487-96 (2013).

66. Yagi, T., Ito, D., Okada, Y., et al. Modeling familial Alzheimer's disease with induced pluripotent stem cells. Hum. Mol. Genet. 20, 4530-9 (2011).

67. ${ }^{* *}$ Shcheglovitov, A., Shcheglovitova, O., Yazawa, M., et al. SHANK3 and IGF1 restore synaptic deficits in neurons from $22 q 13$ deletion syndrome patients. Nature 503, 267-271 (2013). This article performs an analysis of excitatory synaptic transmission in iPSCs delived from Phelan-McDermid syndrome patients and control subjects. The defective transmission in patientderived cells can be rescued by engaging a conserved molecular pathway in these cells.

68. Mullard, A. Stem-cell discovery platforms yield first clinical candidates. Nat. Rev. Drug Discov. 14, 589-591 (2015).

69. Medina, M. \& Avila, J. Further understanding of tau phosphorylation: implications for therapy. Expert Rev. Neurother. 15, 115-22 (2015).

70. Wischik, C. M., Staff, R., Wischik, D., et al. Tau aggregation inhibitor therapy: an exploratory phase 2 study in mild or moderate Alzheimer's disease. J. Alzheimers. Dis. 44, 705-20 (2015).

71. Castillo-Carranza, D. L., Gerson, J., Sengupta, U., et al. Specific targeting of tau oligomers in Htau mice prevents cognitive impairment and tau toxicity following injection with brain-derived tau oligomeric seeds. J. Alzheimers. Dis. 40 Suppl 1, S97-S111 (2014). 
72. Magen, I. \& Gozes, I. Davunetide: Peptide therapeutic in neurological disorders. Curr. Med. Chem. 21, 2591-8 (2014).

73. Kalra, J. \& Khan, A. Reducing $A \beta$ load and tau phosphorylation: Emerging perspective for treating Alzheimer's disease. Eur. J. Pharmacol. 764, 571-81 (2015).

74. Alzforum - Networking for a cure. Available from:

<http://www.alzforum.org/therapeutics> [3 Feb 2016].

75. Morimoto, B. H., Fox, A. W., Stewart, A. J., et al. Davunetide: a review of safety and efficacy data with a focus on neurodegenerative diseases. Expert Rev. Clin. Pharmacol. 6, 483-502 (2013).

76. Boxer, A. L., Lang, A. E., Grossman, M., et al. Davunetide in patients with progressive supranuclear palsy: a randomised, double-blind, placebo-controlled phase 2/3 trial. Lancet Neurol. 13, 676-685 (2014).

77. Iqbal, K., Zaidi, T., Wen, G., et al. Defective Brain Microtubule Assembly in Alzheimer'S Disease. Lancet 328, 421-426 (1986).

78. Lee, V. M., Balin, B. J., Otvos, L., et al. Q. A68: a major subunit of paired helical filaments and derivatized forms of normal Tau. Science 251, 675-678 (1991).

79. Grundke-Iqbal, I. \& Iqbal, K. Neuronal cytoskeleton in the biology of Alzheimer disease. Prog. Clin. Biol. Res. 317, 745-753 (1989).

80. Braak, H. \& Braak, E. Staging of Alzheimer's disease-related neurofibrillary changes. Neurobiol. Aging 16, 271-278 (1995).

81. Bancher, C., Braak, H., Fischer, P., et al. Neuropathological staging of Alzheimer lesions and intellectual status in Alzheimer's and Parkinson's disease patients. Neurosci. Lett. 162, 179-82 (1993).

82. Nelson, P. T., Alafuzoff, I., Bigio, E., et al. Correlation of Alzheimer Disease Neuropathologic Changes With Cognitive Status. J. Neuropathol. Exp. Neurol. 71, 362-381 (2012).

83. Spillantini, M. G., Crowther, R. A. \& Goedert, M. Comparison of the neurofibrillary pathology in Alzheimer's disease and familial presenile dementia with tangles. Acta Neuropathol. 92, 42-48 (1996).

84. Luna-Muñoz, J., Chávez-Macías, L., García-Sierra, F., et al. Earliest stages of tau conformational changes are related to the appearance of a sequence of specific phospho-dependent tau epitopes in Alzheimer's disease. J. Alzheimers. Dis. 12, 365-375 (2007).

85. Nakamura, K., Greenwood, A., Binder, L., et al. Proline Isomer-Specific Antibodies Reveal the Early Pathogenic Tau Conformation in Alzheimer's Disease. Cell 149, 232-244 (2012). 
86. Flores-Rodríguez, P., Ontiveros-Torres M. A., Cárdenas-Aguayo M. C., et al. The relationship between truncation and phosphorylation at the C-terminus of tau protein in the paired helical filaments of Alzheimer's disease. Front.

Neurosci. 9, 33 (2015).

87. Hoppe, J. B., Salbego, C. G. \& Cimarosti, H. SUMOylation: Novel Neuroprotective Approach for Alzheimer's Disease? Aging Dis. 6, 322-330 (2015).

88. Morris, M., Knudsen, G. M., Maeda, S., et al. Tau post-translational modifications in wild-type and human amyloid precursor protein transgenic mice. Nat. Neurosci. 18, 1183-1192 (2015).

89. *Matsuo, E. S., Shin, R., Billingsley, M., et al. Biopsy-derived adult human brain tau is phosphorylated at many of the same sites as Alzheimer's disease paired helical filament tau. Neuron 13, 989-1002 (1994). This report demonstrates that the detection of phosphorylation state of adult human and rodent tau is highly dependent upon rapid processing of biopsy brain.

90. *Seubert, P., Mawal-Dewan, M., Barbour, R., et al. Detection of phosphorylated Ser262 in fetal tau, adult tau, and paired helical filament tau. The Journal of biological chemistry, 270(32)18917-18922 (1995). This report demonstrates that adult human tau is moderately phosphorylated under normal physiological conditions but that the phosphorylation state declines within minutes.

91. *Mudher, A K., Woolley, S. T., Perry, V. H., et al. Induction of hyperphosphorylated tau in living slices of rat hippocampal formation and subsequent detection using an ELISA. J. Neurosci. Methods 88, 15-25 (1999). ). This study shows AT8-positive tau within the somato-dendritic compartment of rapidly processed rat brain slices.

92. Mudher, A. K., Perry, V. H.\& Road, M. Matters arising using okadaic acid as a tool for the in vivo induction of hyperphosphorylated tau. Neuroscience 85, 1329-1332 (1998).

93. Götz, J., Barmettler, R., Ferrari, A., et al. In Vivo Analysis of Wild-type and FTDP-17 Tau. Ann. New York Acad. Sci. 920, 126-133 (2000).

94. Higuchi, M., Trojanowski, J. Q. \& Lee, V. M. Tau protein and tauopathies. Neuropsychopharmacol. Fifth Gener. Prog. 1339-1354 (2002).

95. Lewis, J., McGowan, E., Rockwood, J., et al. Neurofibrillary tangles, amyotrophy and progressive motor disturbance in mice expressing mutant (P301L) tau protein. Nat. Genet. 25, 402-405 (2000).

96. Puzzo, D., Gulisano, W., Palmeri, A., et al. Rodent models for Alzheimer's disease drug discovery. Expert Opin Drug Discov. 10, 703-711 (2015).

97. Ghetti, B., Oblak, A. L., Boeve, B. F., et al. Invited review: Frontotemporal dementia caused by microtubule-associated protein tau gene (MAPT) mutations: 
a chameleon for neuropathology and neuroimaging. Neuropathol. Appl. Neurobiol. 41, 24-46 (2015).

98. Andorfer, C., Kress, Y., Espinoza, M., et al. Hyperphosphorylation and aggregation of tau in mice expressing normal human tau isoforms. $J$. Neurochem. 86, 582-590 (2003).

99. Spillantini, M. G. \& Goedert, M. Tau pathology and neurodegeneration. Lancet. Neurol. 12, 609-622 (2013).

100. Usenovic, M., Niroomand, S., Drolet, R. et al. Internalized Tau Oligomers Cause Neurodegeneration by Inducing Accumulation of Pathogenic Tau in Human Neurons Derived from Induced Pluripotent Stem Cells. J. Neurosci. 35, 1423414250 (2015).

101. Sullivan, S. E. \& Young-Pearse, T. Induced pluripotent stem cells as a discovery tool for Alzheimer's disease. Brain Res. (15)742-748 (2015). 


\section{Figure 1. Using human neurons to improve clinical translation in in dementia}

research. Currently, drug targets for neurodegenerative disease are identified predominantly from investigations with animal models (either in vivo or in vitro) and human post mortem tissue $(A)$. Inadequate disease simulation in these paradigms contributes to the lack of translation towards effective clinical therapies. One way around this is to utilise living human neurons which currently take the form of stem-cell derived iPSC neuron models (B). We propose the wider use of resected human brain tissue (C) should be incorporated into this experimental armoury. In this way, target engagement and mechanistic efficacy can be validated prior to phase II/III clinical trials (D). This will undoubtedly increase the pharmacological confidence in the potential of drugs that get to clinical trials and may improve translation.

\section{Figure 2. Benefits and challenges of using iPSC-derived neural cultures for} novel drug discovery in dementia research. (A) Healthy patient-derived iPSCs committed to neural stem cells and differentiated to mature neurons and astrocytes provide a new tool to test normal human neuronalfunction and some safety aspects of new drugs. (B) Neurons and astrocytes can be obtained in the same manner from individuals with disease-related familial mutations or individuals affected by disease. These cells provide a valuable tool to analyse disease mechanisms and to test some efficacy aspects of new drugs. The challenges (depicted by numbers) in the use of hiPSCs-derived neural cultures detailed in this review include: 1. Variability between patients, where genome wide and epigenetic changes underpin and modify the healthy or disease phenotype. 2. Variability between cell lines, not all the cell lines have the same differentiation potential. 3. Variability within a single hiPSCs line due to passaging and accumulation of mutations. 4. Differentiation and maturation of neurons, incorporation of other cell types (e.g. microglia and endothelium). 
Figure 1:

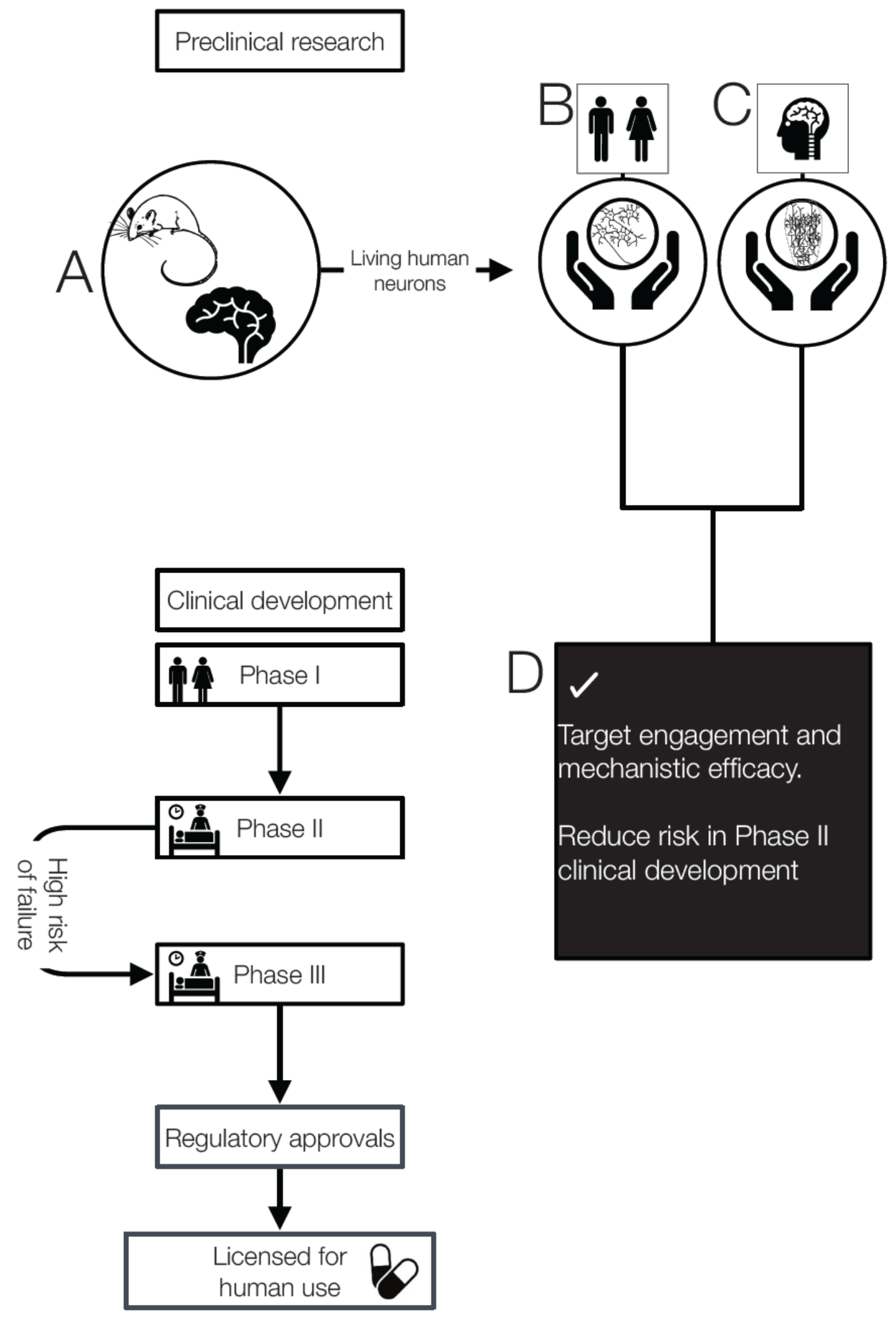


Figure 2:

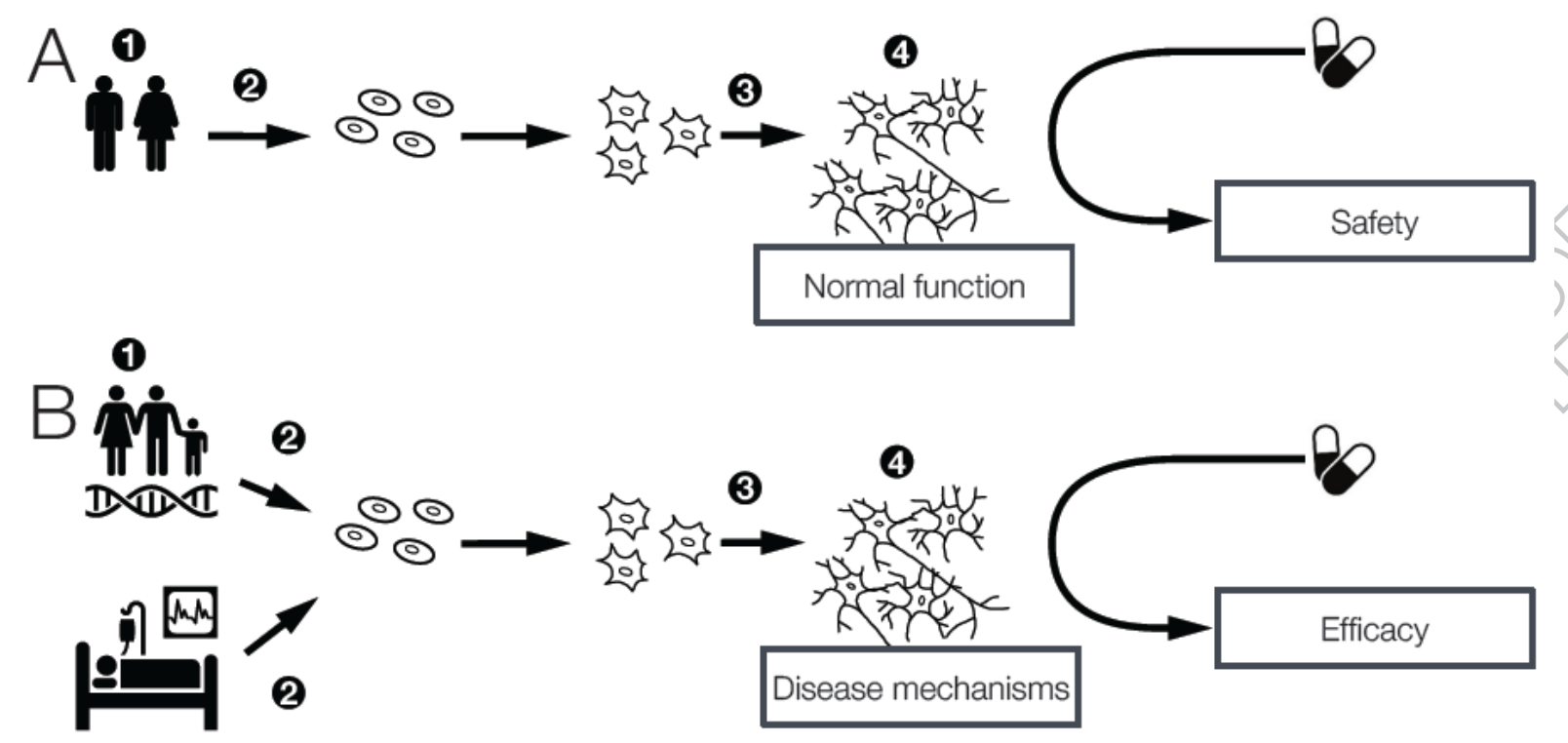

